\section{When culture, traditions and public health clash: A paradigm shift urgently needed to stem the spread of COVID-19 in Zimbabwe}

To the Editor: For almost a year, Zimbabwe has faced the ongoing public health threat of the COVID-19 pandemic. To curb its spread, the government has put in place a myriad of restrictions, including a nationwide lockdown that limits the movement of people, bans social gatherings and led to school closures. ${ }^{[1,2]}$ Studies have reported poor adherence to some of these measures. ${ }^{[3]}$ While economic pressure has been reported to largely contribute to poor adherence in an otherwise informal economy, the effect of some cultural tendencies on the COVID-19 pandemic response should not be ignored. It is widely known that tradition and culture play a pivotal role in determining individual and community-level conduct, such as attributions to illness, health-seeking behaviours and community willingness to comply with measures instituted to curb the spread of disease. ${ }^{[4]}$ Cultural beliefs and values contribute, to a large extent, to the success or failure of the country's efforts to contain the spread of COVID-19.

For instance, funerals can be COVID-19 super-spreader events. ${ }^{[5]}$ Traditional funeral and burial rituals in Zimbabwe, especially in rural areas, usually result in large numbers of family and friends attending to pay their last respects to their loved ones. ${ }^{[6]}$ Further, it is a general expectation that most of the deceased are buried at their rural homes, even if they die in another part of the country. Bodies and mourners, even during COVID-19 lockdown, are transported nicodemously across the country. The government recently banned the transportation of corpses across provinces, ${ }^{[7]}$ but reversed the decision within a few days ${ }^{[8]}$ after a public outcry. Studies have reported poor adherence to COVID-19 guidelines, with the number of funeral attendees exceeding the allowed limit of 30 and lack of social distancing and proper sanitisation arrangements for mourners. Furthermore, traditionally, mourners are expected to shake hands when they meet at a funeral, a culture locally known in Shona (spoken by the majority of Zimbabweans) as kubata maoko. ${ }^{[3]}$ Traditional leaders in rural areas have very little will and capacity to enforce COVID-19 restrictions on mourners. The identification and inclusion of cultural parameters in the national COVID-19 measures, even as an afterthought, are important in the development of an effective pandemic control strategy.

Another example of a cultural norm in Zimbabwe is that relatives and friends are expected to visit the sick. This has the potential to spread COVID-19 in the community, as people tend to gather at the sick person's residence. The traditional marriage ceremony that involves payment of bride price involves large numbers of people gathering to witness the ceremony. Variations in these cultural and traditional practices are prevalent in most countries in southern Africa. ${ }^{[9]}$

In conclusion, we call for expanded health education of the populace for improved understanding of the COVID-19 risks involved. Moreover, there remains a need for co-ordinated responses between public health experts and traditional leaders. There is an urgent need for the resourcing of traditional leaders to effectively contribute to the fight against COVID-19 in their areas. ${ }^{[10]}$ These leaders will be useful in community mobilisation, dispelling pandemic rumours and myths, and pushing for concessions regarding measures incompatible with local cultural norms. Communities should also be encouraged to embrace technology in the form of online meetings for marriage ceremonies and other such activities to avoid in-person gatherings.

\section{Tafadzwa Dzinamarira}

Discipline of Public Health Medicine, School of Nursing and Public Health, College of Health Sciences, University of KwaZulu-Natal, Durban; and ICAP at Columbia University, Pretoria, South Africa anthonydzina@gmail.com

\section{Godfrey Musuka}

ICAP at Columbia University, Harare, Zimbabwe

\footnotetext{
1. Dzobo M, Chitungo I, Dzinamarira T. COVID-19: A perspective for lifting lockdown in Zimbabwe. Pan Afr Med J 2020;35(Suppl 2):13. https://doi.org/10.11604/pamj.2020.35.2.23059

2. Dzinamarira T, Musuka G. The paradox of re-opening schools in Zimbabwe in the COVID-19 era Public Health Pract 2021;2:100070.

3. Musarandega $\mathrm{H}$, Chitongo L. A contextual COVID-19 social distancing monitoring strategy for remote communal settings: Insights from Biriiri communal lands, Zimbabwe. Afr J Govern Develop 2020;9(11):309-328

4. Idriss A, Diaconu K, Zou G, Senesi R, Wurie HR, Witter S. Rural-urban health seeking behaviours for non-communicable diseases in Sierra Leone. BMJ Glob Health 2020;5:e002024. https://doi.org/10.1136/ bmigh-2019-002024

5. Centers for Disease Control. Funeral guidance for individuals and families. 2020. https://www.cdc.gov/ coronavirus/2019-ncov/daily-life-coping/funeral-guidance.html (accessed 16 February 2021).

Saidi U. agonya neiko mfanha uyu? Of death and funerals - a semiotic exploration of the Shona funeral 6. Saidi U. agonya neiko mfanha in ritual in Zimbabwe. Af ldent News24. Zimbabwe bans traditional funerals after spike in Covid cases. https://www.news24.com/ amp/news24/africa/news/zimbabwe-bans-traditional-funerals-after-spike-in-covid-cases-2021011 (accessed 16 February 2021)

8. XinhuaNet. COVID-19: Zimbabwean government reverses ban on movement of bodies. http://www xinhuanet.com/english/2021-01/21/c_139687615.htm (accessed 16 February 2021).

9. Cultural insights. https://www.international.gc.ca/global-affairs-affaires-mondiales/services/cfsi-icse/ cultural-insights-apercu-culturelles/index.aspx?lang=eng (accessed 16 February 2021).

10. Dziva C. Prospects and challenges for traditional leaders in combating COVID-19 pandemic in rural Zimbabwe. Soc Sci Human Open 2020. https://doi.org/10.2139/ssrn.3599770
}

S Afr Med J 2021;111(4):279. https://doi.org/10.7196/SAMJ.2021.v111i4.15637 